\title{
Antibiotic resistance is linked to carriage of papC and iutA virulence genes and phylogenetic group $D$ background in commensal and uropathogenic Escherichia coli from infants and young children
}

\author{
N. Karami ${ }^{1,2}$ • A. E. Wold ${ }^{1}$ I. Adlerberth ${ }^{1}$
}

Received: 23 September 2016 / Accepted: 22 November 2016/Published online: 6 December 2016

(C) The Author(s) 2016. This article is published with open access at Springerlink.com

\begin{abstract}
P fimbriae, enabling adherence to colonic and urinary epithelium, and aerobactin, an iron sequestering system, are both colonization factors in the human colon and virulence factors for urinary tract infection. The colonic microbiota is suggested to be a site suitable for the transfer of antibiotic resistance genes. We investigated whether phenotypic resistance to antibiotics in commensal and uropathogenic Escherichia coli from infants and young children is associated with carriage of virulence genes and to phylogenetic group origin and, in the case of fecal strains, to persistence in the gut and fecal population levels. The commensal strains $(n=272)$ were derived from a birth cohort study, while the urinary isolates $(n=205)$ were derived from outpatient clinics. Each strain was assessed for phenotypic antibiotic resistance and for carriage of virulence genes (fimA, papC, sfaD/E, hlyA, iutA, $k f i C$, and neuB), phylogenetic group (A, B1, B2, or D), and markers of particular virulent clones (CGA-D-ST69, O15:H1-D-ST393, and O25b:H4-B2-ST131). Resistance to ampicillin, tetracycline, and trimethoprim was most prevalent. Multivariate analysis showed that resistance to any antibiotic was significantly associated with carriage of genes encoding $\mathrm{P}$ fimbriae (papC) and aerobactin (iutA), and a phylogenetic group D origin. Neither fecal population numbers nor the capacity for long-term persistence in the gut were related to antibiotic resistance among fecal strains. Our study confirms
\end{abstract}

N. Karami

nahid.karami@microbio.gu.se

1 Department of Infectious Diseases, Institute of Biomedicine, The Sahlgrenska Academy, University of Gothenburg,

Gothenburg, Sweden

2 Department of Clinical Microbiology, University of Gothenburg, Guldhedsgatan 10A, 41346 Göteborg, Sweden the importance of phylogenetic group D origin for antibiotic resistance in $E$. coli and identifies the virulence genes papC and iutA as determinants of antibiotic resistance. The reason for the latter association is currently unclear.

\section{Introduction}

Escherichia coli is the leading cause of urinary tract infection (UTI), which frequently occurs in infants and young children [1]. Strains causing UTI usually originate in the patient's own colonic microbiota [2]. Escherichia coli strains segregate into four major phylogenetic groups, termed A, B1, B2, and D. Most strains causing UTI are found within group B2, and to a lesser extent group $\mathrm{D}$, while most strains belonging to the $\mathrm{A}$ and B1 phylogenetic groups have low pathogenic potential [3]. Further, a majority of uropathogenic $E$. coli strains express $\mathrm{P}$ fimbriae, which mediates adherence to colonic and urinary tract epithelium, the iron sequestering aerobactin system, and certain $\mathrm{O}$ and $\mathrm{K}$ antigens. However, these traits are also associated with superior capacity to colonize the human colon. Thus, E. coli strains that persist for long periods in the commensal microbiota more often than transient strains belong to group B2 [4], have $\mathrm{O}$ and $\mathrm{K}$ antigens characteristic of uropathogenic strains [5], and carry the genes for $\mathrm{P}$ fimbriae and aerobactin $[6,7]$. In a rodent model, $P$ fimbriae and capsule K5 confer the capacity to colonize the intestinal tract, suggesting that known UTI virulence traits in E. coli might primarily serve to increase fitness in the natural niche, the colon $[8,9]$. The colonic microbiota has been suggested to be a milieu that favors transfer of antibiotic resistance genes from resistant to initially susceptible strains, and fitness in the human gut commensal microbiota might enhance the probability of acquisition of resistance genes [10]. Furthermore, colonization of the human gut would increase the likelihood 
of the strains being exposed to antibiotics used in human clinical practice, which would tend to positively select for antibiotic-resistant strains in the microbiota.

Certain uropathogenic E. coli clones are known to have accumulated antibiotic resistance genes, for example, clone O25b:H4-B2-ST131 [11] that frequently carries genes encoding extended-spectrum beta-lactamase (ESBL) of the CTX-M-15 type [12], clone CGA-D-ST69 that is globally spread and often carries genes conferring resistance to ampicillin, chloramphenicol, streptomycin, sulfonamides, tetracycline, and trimethoprim [13, 14], and clone O15:H1-D-ST393 that has acquired various antibiotic resistance genes over the last several decades [15]. The prevalence of these clones among commensal $E$. coli is unknown.

The aim of the present study was to investigate whether resistance to commonly used antibiotics is related to phylogenetic group, markers of virulence, and fitness in the commensal gut microbiota. Fecal and urinary E. coli isolates from Swedish infants were screened for antibiotic resistance and their virulence gene carriage, clonal origin, and phylogenetic group allocation were analyzed. Uropathogenic clones with known accumulation of antibiotic resistance genes were identified. A part of the fecal strains had previously been characterized regarding fecal population counts and capacity for long-term persistence in the microbiota [6]. Our results indicate that certain virulence traits are associated with an antibiotic-resistant phenotype.

\section{Materials and methods}

\section{Commensal fecal $E$. coli strains from Swedish infants}

In total, 272 commensal fecal $E$. coli strains isolated during 1998-2002 from 128 Swedish 0-1-year-old infants included in the birth cohort ALLERGYFLORA were studied. ALLERGYFLORA was designed to investigate the impact of gut microbiota on allergy development and the methodology has been described previously [16]. In brief, rectal swabs obtained on day 3 were cultured semi-quantitatively and fecal samples obtained at $1,2,4$, and 8 weeks and at 6 and 12 months of age were cultured quantitatively on Drigalski agar [16]. Isolates were speciated using the API 20E biotyping system (bioMérieux, Marcy-l'Etoile, France) and E. coli were strain-typed using random amplified polymorphic DNA and analyzed regarding virulence gene carriage and phylogenetic group allocation $[4,6]$. In each fecal sample, the counts of each strain [colony-forming units $(\mathrm{CFU}) / \mathrm{g}$ feces] was determined [16]. In addition, for 70 of the 128 infants, each strain has previously been characterized as resident or transient in the microbiota of the infant, the former defined as colonizing for at least 3 weeks, the latter for a shorter period [17], and the
E. coli colonization pattern of these 70 infants has been reported [6].

Each of the 272 strains identified were included only once in each analysis, even if it was isolated from an infant on several sampling occasions.

\section{Urinary E. coli isolates from Swedish infants}

A total of 205 community-acquired urinary $E$. coli isolates from 0-2-year-old children sampled in November 2002February 2003 or March 2004-August 2005 were included. Consecutive urinary samples cultured at the Clinical Microbiology Laboratory, Sahlgrenska University Hospital and yielding $E$. coli as the primary pathogen were included providing that: (1) they were derived from pediatric outpatients' clinics or the emergency ward at the Queen Silvia Children's Hospital in Gothenburg or (2) were from children aged 0-2 years old who had not yielded a previous positive urinary culture or been hospitalized prior to the positive sample. Isolation and speciation of $E$. coli were done using routine methods. For this study, each speciation was confirmed by using API 20E biotyping (bioMérieux).

\section{Virulence genotyping, phylogenetic group typing, and clonal identification}

All urinary isolates and 129 fecal E. coli strains that had previously not been analyzed were screened for carriage of fimA, papC, sfaD/E, neuB, kfiC, iutA, and $h l y A$, as previously described [6].

Assignment to one of the phylogenetic groups A, B1, B2, or $\mathrm{D}$ was done using a previously described triplex polymerase chain reaction (PCR) method [18].

All phylogenetic group D strains were screened for the clones CGA-D-ST69 and O15:H1-D-ST393 [19-21]. Phylogenetic group B2 strains were screened to identify the O25b:H4-B2-ST131 clone [22]. The E. coli strains CCUG 55212, CCUG 41424, and CCUG 61908 were used as reference strains.

\section{Antibiotic susceptibility}

Phenotypic resistance to antibiotics was assessed using the agar disk diffusion method as described by the Nordic Committee on Antimicrobial Susceptibility Testing (NordicAST; http://www.nordicast.org). The following antibiotic disks were used: ampicillin, cefuroxime, cefoxitin, mecillinam, cefadroxil, ceftazidime, chloramphenicol, gentamicin, tobramycin, nitrofurantoin, nalidixic acid, tetracycline, and trimethoprim (Oxoid Ltd., Hants, UK). Strains belonging to the CGA-D-ST69 clone were also tested using disks containing streptomycin and sulfonamide. The antibiotic disks were applied on agar plates seeded with 
bacteria and the plates were incubated aerobically at $37^{\circ} \mathrm{C}$ for 16-24 h. Thereafter, the zone diameters were measured and the strains were defined as sensitive or resistant according to the breakpoints provided by NordicAST.

At least the first isolate of each strain $(n=272)$ was tested for phenotypic resistance to antibiotics, and the resistance pattern of the first isolate of each strain was used in the analyses.

\section{Statistical methods}

Proportions were compared using Fisher's exact test (GraphPad Prism; GraphPad Software, La Jolla, CA, USA). The data were analyzed by multiple linear regression (SPSS version 21 for Windows; SPSS Inc., Chicago, IL, USA) to assess the independent association of different factors with antibiotic resistance in E. coli.

\section{Results}

\section{Antibiotic resistance in fecal and urinary $E$. coli strains}

Twenty-one percent of the commensal fecal strains and $40 \%$ of the urinary strains were resistant to at least one antibiotic $(p<0.0001)$. Resistance against ampicillin, tetracycline, and trimethoprim were most prevalent and more common among UTI than fecal strains ( $p \leq 0.002$, Table 1$)$.

Urinary strains were isolated somewhat later (2002-2005) than the fecal strains (1998-2002). However, resistance rates in neither of these strain collections increased over the sampling period (data not shown). Furthermore, urinary strains isolated in $2002(n=63)$ were significantly more resistant than fecal strains collected during this year $(n=20)(p=0.007)$. Another potential source of confounding was that commensal strains were isolated from 0-1-year-old infants, while urinary strains derived from 0-2-year-old infants. However, when comparing commensal strains isolated from $0-1$-year-old infants with UTI isolates from infants of the same age, resistance was significantly higher in UTI isolates than commensal strains, 21 vs. $42 \%, p<0.0001$. Further, resistance among UTI isolates was not significantly different between isolates from 0-1-year-old infants compared with those from 1-2year-old infants, 42 vs. $31 \%, p=0.26$.

\section{Antibiotic resistance and phylogenetic lineages}

Uropathogenic E. coli strains mostly belong to phylogenetic groups B2 or D. Accordingly, a larger fraction of urinary compared to fecal strains belonged to phylogenetic groups B2 (72 vs. $46 \%, p<0.0001)$ and D (20 vs. $14 \%, p=0.11)$. Instead, more fecal than urinary strains belonged to phylogenetic groups A (28 vs. $4 \%, p<0.0001)$ and B1 (12 vs. $4 \%$, $p=0.002)$.

Resistance was more common among urinary than among fecal strains within each phylogenetic group, the difference being highly significant for groups B2 and D strains ( $p=0.009$ and $p=0.003$, respectively) and of borderline significance for groups B1 and A strains ( $p=0.05$ for both). Furthermore, regardless of clinical origin, group D strains were significantly more often resistant than groups B2 and B1 strains (urinary strains: $p<0.0001, p=0.04$; fecal strains: $p=0.03, p=0.004$ ) (Fig. 1a).
Table 1 Prevalence of resistance to antibiotics among fecal and urinary Escherichia coli strains deriving from Swedish infants

\begin{tabular}{|c|c|c|c|c|c|}
\hline & \multicolumn{5}{|c|}{ Resistance prevalence } \\
\hline & \multicolumn{2}{|c|}{ Fecal strains $(n=272)$} & \multicolumn{2}{|c|}{ Urinary isolates $(n=205)$} & \multirow[t]{2}{*}{$p$-Value } \\
\hline & $n$ & $\%$ & $n$ & $\%$ & \\
\hline Ampicillin & 32 & 12 & 60 & 29 & $<0.0001$ \\
\hline Tetracycline & 28 & 10 & 42 & 20 & 0.002 \\
\hline Trimethoprim & 21 & 8 & 38 & 19 & 0.0006 \\
\hline Chloramphenicol & 3 & 1 & 17 & 8 & 0.0001 \\
\hline Nalidixic acid & 2 & 1 & 6 & 3 & 0.08 \\
\hline Nitrofurantoin & 1 & 0.5 & 0 & 0 & \\
\hline Gentamicin & 0 & 0 & 2 & 1 & \\
\hline Mecillinam & 0 & 0 & 1 & 0.5 & \\
\hline Any of the above antibiotics & 57 & 21 & 82 & 40 & $<0.0001$ \\
\hline
\end{tabular}

Fecal E. coli strains derived from 128 infants followed during the first year of life with regular fecal sampling. Individual strains were identified by random amplified polymorphic DNA [6]. UTI E. coli isolates were consecutive isolates obtained from positive urinary samples of children below 2 years of age presenting with UTI at pediatric outpatient clinics or the emergency ward at the regional children's hospital. All isolates were tested by disk diffusion for resistance to the following antibiotics: ampicillin, cefuroxime, cefoxitin, mecillinam, cefadroxil, ceftazidime, chloramphenicol, gentamicin, tobramycin, nitrofurantoin, nalidixic acid, tetracycline, and trimethoprim. Only $p$-values below 0.10 are presented (Fisher's exact test) 


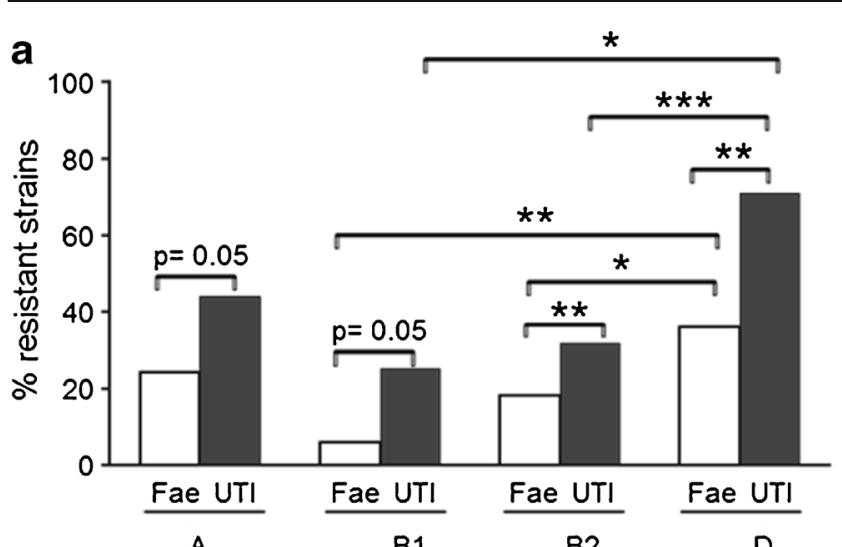

A

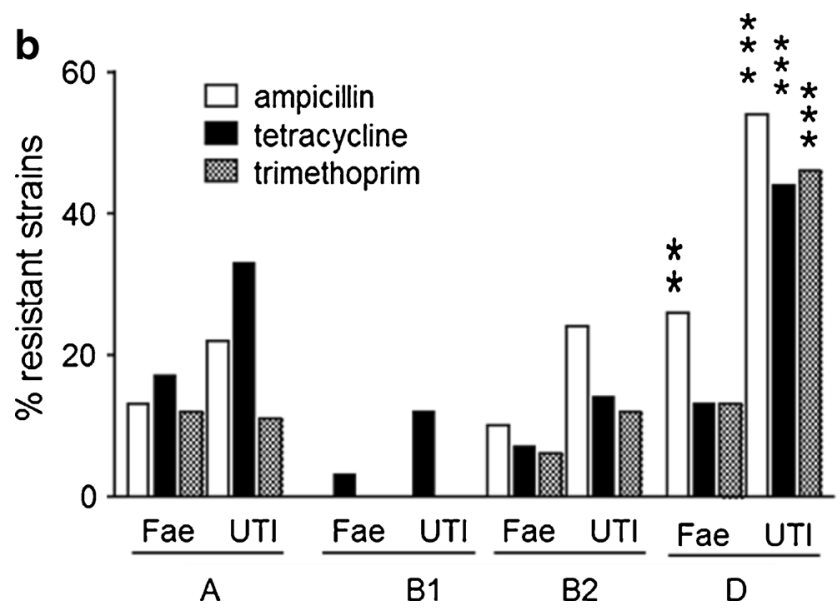

Fig. 1 Antibiotic resistance in relation to phylogenetic group origin. a Prevalence of resistance to any of the screened antibiotics among fecal (Fae) and urinary (UTI) isolates as a function of phylogenetic group. b Resistance to ampicillin (white bars), tetracycline (black bars), and trimethoprim (checkered bars) as a function of phylogenetic group and clinical origin. The asterisks denote significance comparing the resistance of group D fecal or urinary strains to the other phylogenetic groups combined (Fisher's exact test)

Figure $1 \mathrm{~b}$ shows resistance to individual antibiotics in relation to phylogenetic group origin. Urinary group D strains were most often resistant to each of the antibiotics $(p \leq 0.0001$ for each, compared to urinary strains of the other groups combined) and fecal group D strains were more often resistant to ampicillin than fecal strains of the other phylogenetic groups (26 vs. $9 \%, p=0.004)$.

As evident from Fig. 1b, ampicillin resistance was the most common resistance type in groups B2 and D strains, regardless of origin, while groups A and B1 strains were more often resistant to tetracycline than the other two antibiotics. Hence, resistance to ampicillin was significantly more common in strains belonging to groups B2 and D (combined) than in strains belonging to groups A and B1 (combined), 23 vs. $11 \%, p=0.001$. In contrast, resistance to tetracycline was equally common in strains from phylogenetic groups $\mathrm{A}$ and B1 (combined) and from phylogenetic groups B2 and D (combined) (15 vs. 17\%, NS).

\section{Antibiotic resistance and virulence factor genes}

UTI strains more often than fecal strains carried all tested virulence genes, i.e., fimA ( 74 vs. $73 \%, p=0.92)$, papC (72 vs. $29 \%, p<0.0001$ ), sfaD/E (45 vs. $27 \%, p<0.0001$ ), hlyA ( 45 vs. $22 \%, p<0.0001$ ), iutA ( 56 vs. $28 \%, p<0.0001$ ), neuB ( 31 vs. $22 \%, p=0.03)$, and $k f i C(15$ vs. $7 \%, p=0.009)$. Furthermore, the two virulence factors $p a p C$ and iutA were both significantly more common among UTI strains as compared with fecal strains within the same phylogenetic group (papC: $p<0.0001$, iutA: $p=0.007$ in group B2 strains; papC: $p=0.0009$, iutA: $p<0.0001$ in group D strains).

The relation between antibiotic resistance and virulence gene carriage is shown in Fig. 2. In both fecal (Fig. 2a) and UTI strains (Fig. 2b), strains carrying papC or iutA were more often resistant to at least one of the tested antibiotics than were strains lacking these traits. Figure $2 \mathrm{c}-\mathrm{f}$ shows the association between antibiotic resistance and virulence gene carriage within each phylogenetic group, including both fecal and urinary strains in the analyses. Carriage of $p a p C$ was significantly more common among resistant than susceptible strains within phylogenetic groups A (Fig. 2c), B2 (Fig. 2e), and D (Fig. 2f). Further, carriage of the aerobactin gene, iutA, was significantly more common in resistant than susceptible strains within the A, B1, and D phylogenetic groups (Fig. 2c, d, f).

\section{Prevalence of the CGA-D-ST69, O15:H1-D-ST393, and $\mathrm{O} 25 \mathrm{~b}: \mathrm{H} 4-\mathrm{B} 2-\mathrm{ST} 131$ clones}

Among urinary E. coli, 24 strains (12\%) belonged to the CGA-D-ST69 clone, of which more than $75 \%$ carried iutA and papC. Among the fecal strains, $3(1 \%)$ belonged to this clone, two of which carried fimA, papC, and iutA, and the third one only fimA. Fifty percent of the urinary CGA-D-ST69 isolates were resistant to at least three antibiotics, i.e., ampicillin (58\%), tetracycline (58\%), and trimethoprim (54\%). Among the fecal CGA-D-ST69 strains, two were resistant to ampicillin and trimethoprim, and one was susceptible to all the antibiotics tested. Resistance to at least one of the screened antibiotics was significantly more common among urinary CGAD-ST69 strains than among other urinary strains ( 75 vs. 35\%, $p=0.0003$ ). As this clone is known to be resistant to sulfonamide and streptomycin, we tested resistance to these antibiotics and found 63 and $38 \%$ to be resistant, respectively [14].

Only one $(0.5 \%)$ of the urinary strains and two $(1 \%)$ of the fecal strains belonged to the O15:H1-D-ST393 clone. All carried the iutA gene, and two carried papC. Two of three strains were each resistant to nalidixic acid and tetracycline, and the third fecal strain acquired a resistance plasmid during its colonization in an infant's gut, as described elsewhere [10].

The O25b:H4-B2-ST131 clone that belongs to phylogenetic group B2 was represented by two urinary strains $(1.0 \%)$ and 
Fig. 2 Antibiotic resistance in relation to virulence gene carriage. The prevalence $(\%$ of isolates) of resistance to at least one of the screened antibiotics is shown in isolates with (black bars) or without (white bars) seven virulence genes among fecal (a) and urinary (b) E. coli strains or in strains belonging to the four phylogenetic groups (cf); in this case, both urinary and fecal strains were included. $* p<0.05, * * p<0.01$, and $* * * p<0.001$ (Fisher's exact test)
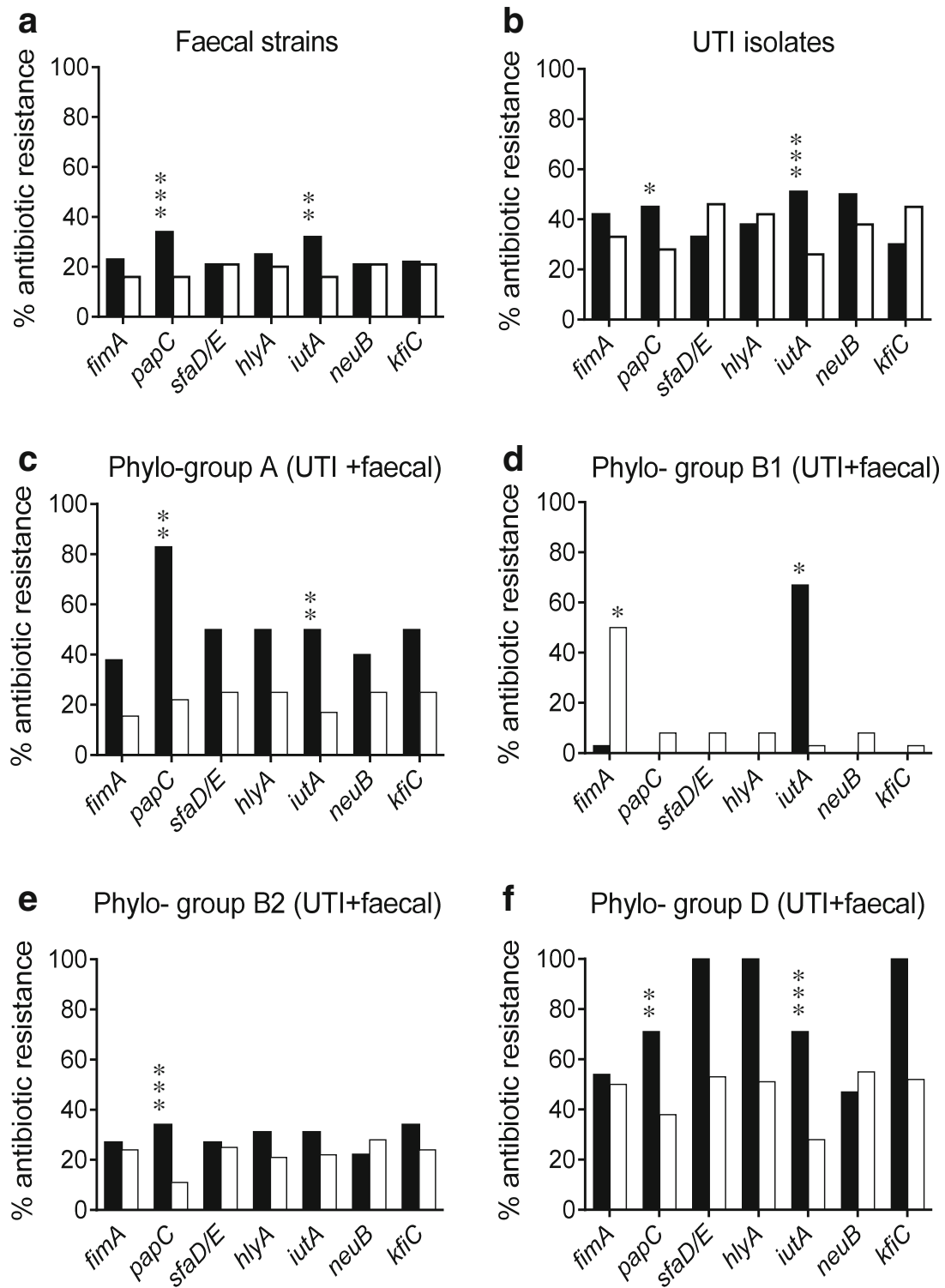

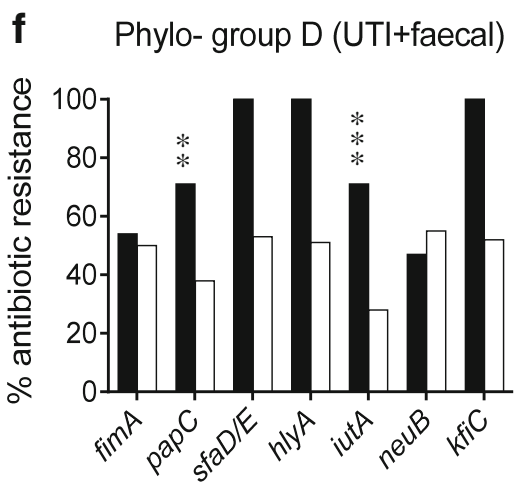

one fecal $(0.4 \%)$ strain. All three carried iutA but were fully susceptible to all the antibiotics tested.

\section{Multiple regression analysis}

As seen in Table 2, phylogenetic group D origin was a strong independent predictor of being resistant to any of the screened antibiotics, as well as for resistance to ampicillin or trimethoprim. Carriage of papC and iutA were also strong predictors for resistance to any antibiotic, $p a p C$ was also an independent predictor for resistance to ampicillin, and iutA was an independent predictor for resistance to both ampicillin and tetracycline. UTI origin was not an independent explanatory factor for antibiotic resistance. CGA-D-ST69 clonal origin was significantly associated with resistance to tetracycline $(p=0.03)$ and tended to be associated with trimethoprim resistance $(p=0.07$, Table 2$)$.

\section{Relation of resistance to population levels and time of persistence of $E$. coli strains in the microbiota}

Figure 3 shows the population levels at different time points after birth of strains that were resistant to at least one antibiotic and fully susceptible strains. At each time point, population counts for all strains that were isolated for the first time at that particular time point were included in the analysis (Fig. 3). There were no significant differences in population levels between resistant and fully susceptible strains on any sampling occasion. Because of the low number of strains resistant to ampicillin, tetracycline, or trimethoprim at each individual time point, the comparison to susceptible strains was not performed for strains resistant to individual antibiotics.

A part of the fecal strains had previously been characterized with respect to their period of colonization in the commensal microbiota [6]. Resident strains often belong to phylogenetic 
Table 2 Multiple regression analysis examining phylogenetic group, clinical origin, and virulence as determinants of resistance to any antibiotic, ampicillin resistance, tetracycline resistance, or trimethoprim resistance

\begin{tabular}{|c|c|c|c|c|c|c|c|c|}
\hline & \multicolumn{8}{|c|}{ Resistance to: } \\
\hline & \multicolumn{2}{|c|}{ Any antibiotic ${ }^{\mathrm{a}}$} & \multicolumn{2}{|c|}{ Ampicillin } & \multicolumn{2}{|c|}{ Tetracycline } & \multicolumn{2}{|c|}{ Trimethoprim } \\
\hline & $\mathrm{B}^{\mathrm{b}}$ & $p$-Value ${ }^{\mathrm{c}}$ & $\mathrm{B}^{\mathrm{b}}$ & $p$-Value ${ }^{c}$ & $\mathrm{~B}^{\mathrm{b}}$ & $p$-Value ${ }^{\mathrm{c}}$ & $\mathrm{B}^{\mathrm{b}}$ & $p$-Value $^{\mathrm{c}}$ \\
\hline Intercept $^{\mathrm{d}}$ & -1.53 & & -0.77 & & -0.30 & & -0.33 & \\
\hline Group D & 1.02 & 0.002 & 1.04 & 0.004 & 0.36 & 0.39 & 0.90 & 0.03 \\
\hline papC & 0.87 & 0.0001 & 0.78 & 0.006 & 0.47 & 0.88 & 0.36 & 0.29 \\
\hline iutA & 0.74 & 0.001 & 0.63 & 0.02 & 0.98 & 0.001 & 0.62 & 0.06 \\
\hline UTI origin & 0.30 & 0.23 & 0.57 & 0.05 & 0.34 & 0.28 & 0.45 & 0.19 \\
\hline CGA-D-ST69 & 0.66 & 0.23 & 0.48 & 0.36 & 1.23 & 0.03 & 1.01 & 0.07 \\
\hline
\end{tabular}

${ }^{a}$ Resistance to the following antibiotics were included in the screening: ampicillin, cefuroxime, cefoxitin, mecillinam, cefadroxil, ceftazidime, chloramphenicol, gentamicin, tobramycin, nitrofurantoin, nalidixic acid, tetracycline, and trimethoprim

${ }^{\mathrm{b}} \mathrm{B}$ : standardized regression coefficient

${ }^{\mathrm{c}}$ Bold $p$-value $\geq 0.05$, statistically significant association

${ }^{\mathrm{d}}$ Intercept: the point at which the curve intersects the $y$-axis group B2 [4] and carry the papC and iutA operons [6, 7]. We included these factors, as well as a phylogenetic group D origin, as explanatory factors in a multiple regression analysis, to determine their contribution to antibiotic resistance. As shown in Table 3, being resident in the microbiota did not contribute to being resistant among the examined fecal strains. Belonging to group B2 was a significant negative predictor of antibiotic resistance, while papC was a significant positive predictor (Table 3).

\section{Discussion}

In the present study, we investigated determinants of resistance to commonly used antibiotics in fecal and urinary

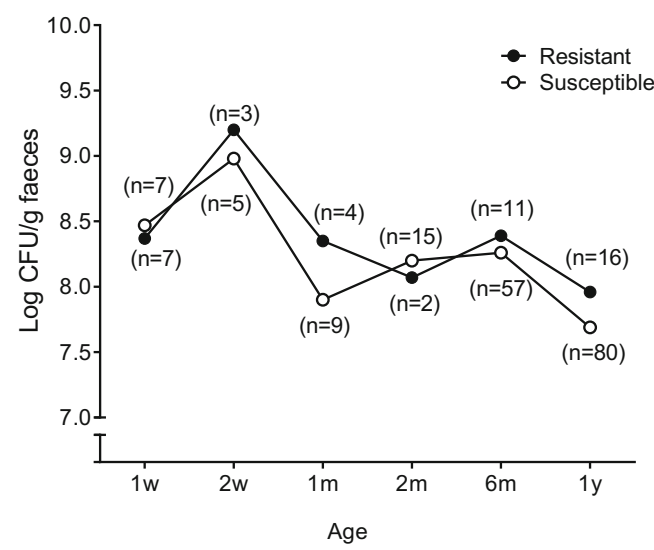

Fig. 3 Fecal population counts of resistant and fully susceptible E. coli strains in infants' samples. Strains exhibiting resistance to at least one antibiotic (filled circles) or strains susceptible to all screened antibiotics (open circles) derived from infants followed with quantitative stool cultures from 1 week to 1 year of age. The average counts of all resistant and susceptible strains that were isolated for the first time at each time point are presented. The numbers within parentheses denote the number of strains represented on each sampling occasion
E. coli strains from Swedish infants and young children below 2 years of age, and examined the relation between antibiotic resistance, virulence gene carriage, and phylogenetic group origin of the strains. In addition, we screened the strains to identify three known uropathogenic clones, i.e., CGA-DST69, O15:H1-D-ST393, and O25b:H4-B2-ST131, to determine if these clones contributed to the pool of antibioticresistant E. coli colonizing and infecting Swedish infants. In the multivariate analysis, we found three factors that contributed independently to the strains being resistant to at least one

Table 3 Multiple regression analysis examining the role of phylogenetic groups, virulence genes, and capacity to persist in the microbiota as determinants of antibiotic resistance among fecal commensal $E$. coli strains from infants

\begin{tabular}{lcc}
\hline & \multicolumn{2}{l}{ Resistance $^{\mathrm{a}}$} \\
\cline { 2 - 3 } & $\mathrm{B}^{\mathrm{b}}$ & $p$-Value \\
\hline Intercept $^{\mathrm{d}}$ & 0.10 & \\
Phylogenetic group D $_{\text {Phylogenetic group B2 }}$ & 0.5 & 0.42 \\
papC & -1.3 & 0.04 \\
iutA & 0.65 & 0.04 \\
Resident strains & 0.71 & 0.12 \\
\hline
\end{tabular}

${ }^{\mathrm{a}}$ Resistance to any of the antibiotics listed in Tables 1 and 2 was the dependent variable

${ }^{\mathrm{b}} \mathrm{B}$ : standardized regression coefficient

${ }^{\mathrm{c}}$ Bold $p$-value $\geq 0.05$, statistically significant association

${ }^{\mathrm{d}}$ Intercept: the point at which the curve intersects the $y$-axis

${ }^{\mathrm{e}}$ Ninety-five resident and 22 transient fecal $E$. coli strains were included in the analysis. Resident strains were those isolated repeatedly from an infant over a period of at least 3 weeks, while transient strains colonized for less than 3 weeks 
of the screened antibiotics; belonging to phylogenetic group $\mathrm{D}$, and carrying the two UTI virulence genes, pap $C$ encoding $\mathrm{P}$ fimbriae and iutA encoding aerobactin. Neither persistence in the microbiota nor attaining high fecal population counts were associated with antibiotic resistance among the fecal strains.

Among the tested antibiotics, resistance to ampicillin, tetracycline, or trimethoprim were most common, but the prevalence was moderate compared to the reported figures from many other countries during the same period [23, 24]. This is in agreement with a generally low resistance rate among Swedish clinical isolates observed in surveillance studies (http://www.ecdc.europa.eu). Still, the $21 \%$ resistance rate among fecal commensal strains found here is considerably higher than the $14 \%$ reported in a Swedish study from the 1970s [25].

Resistance to tested antibiotics was significantly more common among urinary than among fecal strains. This is in accordance with other studies, showing that the frequency of resistance in commensal $E$. coli is lower but correlated to the resistance rate in clinical isolates from the same area [26]. However, clinical origin as a factor associated with resistance disappeared in the multivariate analysis, indicating that phylogenetic group origin and virulence profile were the primary explanatory factors. It is well known that papC and iutA are accumulated in uropathogenic strains.

One factor promoting antibiotic resistance development would be a pronounced capacity to pick up resistance elements transferred horizontally. Phylogenetic group D origin was a significant independent determinant of antibiotic resistance, in agreement with previous studies showing that a group D phylogenetic background per se facilitates acquisition of resistance genes $[27,28]$. In accordance, we have reported on a case of transfer of a resistance plasmid from one group D strain to another in the intestinal microbiota of a child, in relation to treatment with ampicillin, to which the donor strain was resistant [10].

CGA-D-ST69 is a clone that often exhibits multiresistance due to a large conjugative plasmid containing several resistance genes [13]. The CGA-D-ST69 isolates were the most resistant ones, although fecal CGA-D-ST69 isolates were less resistant than urinary isolates of this clone. A CGA-D-ST69 clonal origin was an independent explanatory factor for resistance to tetracycline and tended to be an explanatory factor for resistance to trimethoprim. Notably, only $1 \%$ of the fecal strains, but $12 \%$ of the urinary isolates, belonged to this clone.

Almost $50 \%$ of the infantile fecal E. coli strains examined here belonged to phylogenetic group B2, a figure that we have reported previously [4]. Escherichia coli group B2 strains are the most pathogenic, but had overall low antibiotic resistance both in the urinary isolate collection and among the fecal strains. Indeed, a phylogenetic group B2 origin was a significant negative predictor of antibiotic resistance among fecal strains in the present study. The fact that B2 strains are less likely to carry resistance genes compared to group D strains confirms observations from previous studies [29]. Group B2 strains may be less prone to acquiring plasmids than group D strains. Accordingly, virulence genes in group B2 strains are mostly carried on pathogenicity islands located in the chromosome, whereas group D strains often carry virulence genes, e.g., iutA, on plasmids [29]. However, group B2 is highly genetically diverse, comprising at least nine subgroups [30], some of which may be better at incorporating horizontally transferred genetic elements than others. One of the most pathogenic clones of phylogenetic group B2 is O25b:H4-B2ST131, which is enriched in virulence factor genes and, nowadays, frequently carries resistance genes encoding, e.g., CTX-M, KPC, or NDM enzymes [31]. Co-selection of resistance genes, e.g., CTX-M, and virulence genes in plasmids of the IncFII group was suggested to be of importance for the worldwide dissemination of this clone [32]. However, the three strains of this clone identified in the present study were sensitive to all the antibiotics tested. Our low isolation rate and lack of resistance among ST131 isolates likely reflect a low prevalence of this clone in Sweden before 2005, and an overall low resistance rate in $E$. coli. The prevalence of ESBLproducing $E$. coli was only $0.2 \%$ among urine isolates in 2004 in Western Sweden and only 10\% of these isolates belonged to the ST131 clone [33].

Carriage of either of the virulence genes papC and iutA were independent explanatory factors for antibiotic resistance, including any resistance or resistance to ampicillin. iutA was also an explanatory factor for tetracycline resistance. The pap operon encodes $\mathrm{P}$ fimbriae, which enables adherence to both urinary and colonic epithelial cells and favors both persistence in the gut microbiota and urinary tract virulence [6, 7]. Aerobactin production, encoded by the iutA operon, is also linked to both urinary virulence [34] and persistence in the commensal gut microbiota [7]. Long-term persistence in the microbiota could enhance the chances of acquiring resistance genes and high population counts could also favor gene transfer. However, we could not demonstrate any independent contribution of long-term persistence in the microbiota on antibiotic resistance, nor did we observe any relation between fecal population counts and resistance.

Possibly, resistance genes and virulence genes occur together on transposons or plasmids and are, therefore, co-selected. Carriage of the iutA gene has previously been associated with antibiotic resistance in $E$. coli of extraintestinal origin [34] and is also common among strains producing ESBLs of the CTX-M types [35, 36]. In previous studies, we observed positive associations between carriage of tet genes encoding tetracycline resistance and the virulence genes papC and iutA, as well as between carriage of beta-lactamase genes and $p a p C$ in commensal $E$. coli strains [37, 38]. In contrast, carriage of pap and hly genes is inversely related to resistance against 
fluoroquinolones in E. coli, possibly relating to a partial loss of genomic pathogenicity islands as a consequence of resistance mutations in genes encoding topoisomerases II and IV [39]. As few strains in our material were resistant to quinolones, this potential association could not be examined here.

In conclusion, we confirmed that strains belonging to phylogenetic group D have a special propensity to be resistant to antibiotics and showed that carriage of the virulence genes papC and iutA were also linked to resistance. The reason for the latter observation remains to be explained.

Acknowledgments The authors wish to thank the personnel at the Clinical Microbiology Laboratory, Sahlgrenska University Hospital for their technical assistance, particularly Johan Widjestam for the valuable technical advice. We thank F.L. Nowrouzian for the critical reading of the manuscript.

\section{Compliance with ethical standards}

Funding This work was supported by grants from the Swedish Medical Research Council (no. K98-06X-12612-01A), the Swedish Strategic Programme for the Rational Use of Antimicrobial Agents and Surveillance of Resistance, the Capio Research Foundation, Wilhelm and Martina Lundgren Foundation, and Västra Götaland Region projects (ALFGBG-11574, ALFGBG-210591).

Conflict of interest The authors declare that they have no conflict of interest.

Open Access This article is distributed under the terms of the Creative Commons Attribution 4.0 International License (http:// creativecommons.org/licenses/by/4.0/), which permits unrestricted use, distribution, and reproduction in any medium, provided you give appropriate credit to the original author(s) and the source, provide a link to the Creative Commons license, and indicate if changes were made.

\section{References}

1. Beetz R (2012) Evaluation and management of urinary tract infections in the neonate. Curr Opin Pediatr 24(2):205-211

2. Moreno E, Andreu A, Pigrau C, Kuskowski MA, Johnson JR, Prats G (2008) Relationship between Escherichia coli strains causing acute cystitis in women and the fecal E. coli population of the host. J Clin Microbiol 46(8):2529-2534

3. Johnson JR, Scheutz F, Ulleryd P, Kuskowski MA, O'Bryan TT, Sandberg T (2005) Phylogenetic and pathotypic comparison of concurrent urine and rectal Escherichia coli isolates from men with febrile urinary tract infection. J Clin Microbiol 43(8):3895-3900

4. Nowrouzian FL, Wold AE, Adlerberth I (2005) Escherichia coli strains belonging to phylogenetic group $\mathrm{B} 2$ have superior capacity to persist in the intestinal microflora of infants. J Infect Dis 191(7):1078-1083

5. Wold AE, Caugant DA, Lidin-Janson G, de Man P, Svanborg C (1992) Resident colonic Escherichia coli strains frequently display uropathogenic characteristics. J Infect Dis 165(1):46-52

6. Nowrouzian F, Hesselmar B, Saalman R, Strannegård IL, Aberg N, Wold AE, Adlerberth I (2003) Escherichia coli in infants' intestinal microflora: colonization rate, strain turnover, and virulence gene carriage. Pediatr Res 54(1):8-14
7. Nowrouzian FL, Adlerberth I, Wold AE (2006) Enhanced persistence in the colonic microbiota of Escherichia coli strains belonging to phylogenetic group B2: role of virulence factors and adherence to colonic cells. Microbes Infect 8(3):834-840

8. Herías MV, Midtvedt T, Hanson LA, Wold AE (1995) Role of Escherichia coli $\mathrm{P}$ fimbriae in intestinal colonization in gnotobiotic rats. Infect Immun 63(12):4781-4789

9. Hérias MV, Midtvedt T, Hanson LA, Wold AE (1997) Escherichia coli $\mathrm{K} 5$ capsule expression enhances colonization of the large intestine in the gnotobiotic rat. Infect Immun 65(2):531-536

10. Karami N, Martner A, Enne VI, Swerkersson S, Adlerberth I, Wold AE (2007) Transfer of an ampicillin resistance gene between two Escherichia coli strains in the bowel microbiota of an infant treated with antibiotics. J Antimicrob Chemother 60(5):1142-1145

11. Riley LW (2014) Pandemic lineages of extraintestinal pathogenic Escherichia coli. Clin Microbiol Infect 20(5):380-390

12. Nicolas-Chanoine MH, Blanco J, Leflon-Guibout V, Demarty R, Alonso MP, Caniça MM, Park YJ, Lavigne JP, Pitout J, Johnson JR (2008) Intercontinental emergence of Escherichia coli clone O25:H4 ST131 producing CTX-M-15. J Antimicrob Chemother 61(2):273-281

13. Manges AR, Johnson JR, Foxman B, O'Bryan TT, Fullerton KE, Riley LW (2001) Widespread distribution of urinary tract infections caused by a multidrug-resistant Escherichia coli clonal group. $\mathrm{N}$ Engl J Med 345(14):1007-1013

14. Tartof SY, Solberg OD, Manges AR, Riley LW (2005) Analysis of a uropathogenic Escherichia coli clonal group by multilocus sequence typing. J Clin Microbiol 43(12):5860-5864

15. Olesen B, Scheutz F, Menard M, Skov MN, Kolmos HJ, Kuskowski MA, Johnson JR (2009) Three-decade epidemiological analysis of Escherichia coli O15:K52:H1. J Clin Microbiol 47(6):1857-1862

16. Adlerberth I, Lindberg E, Aberg N, Hesselmar B, Saalman R, Strannegård IL, Wold AE (2006) Reduced enterobacterial and increased staphylococcal colonization of the infantile bowel: an effect of hygienic lifestyle? Pediatr Res 59(1):96-101

17. Sears HJ, Janes H, Saloum R, Brownlee I, Lamoreaux LF (1956) Persistence of individual strains of Escherichia coli in man and dog under varying conditions. J Bacteriol 71:370-372

18. Clermont O, Bonacorsi S, Bingen E (2000) Rapid and simple determination of the Escherichia coli phylogenetic group. Appl Environ Microbiol 66(10):4555-4558

19. Johnson JR, Menard M, Johnston B, Kuskowski MA, Nichol K, Zhanel GG (2009) Epidemic clonal groups of Escherichia coli as a cause of antimicrobial-resistant urinary tract infections in Canada, 2002 to 2004. Antimicrob Agents Chemother 53(7):2733-2739

20. Johnson JR, Owens K, Manges AR, Riley LW (2004) Rapid and specific detection of Escherichia coli clonal group A by genespecific PCR. J Clin Microbiol 42(6):2618-2622

21. Johnson JR, Owens K, Sabate M, Prats G (2004) Rapid and specific detection of the O15:K52:H1 clonal group of Escherichia coli by gene-specific PCR. J Clin Microbiol 42(8):3841-3843

22. Clermont O, Dhanji H, Upton M, Gibreel T, Fox A, Boyd D, Mulvey MR, Nordmann P, Ruppé E, Sarthou JL, Frank T, Vimont S, Arlet G, Branger C, Woodford N, Denamur E (2009) Rapid detection of the O25b-ST131 clone of Escherichia coli encompassing the CTX-M-15-producing strains. J Antimicrob Chemother 64(2):274-277

23. Calva JJ, Sifuentes-Osornio J, Cerón C (1996) Antimicrobial resistance in fecal flora: longitudinal community-based surveillance of children from urban Mexico. Antimicrob Agents Chemother 40(7):1699-1702

24. Domínguez E, Zarazaga M, Sáenz Y, Briñas L, Torres C (2002) Mechanisms of antibiotic resistance in Escherichia coli isolates obtained from healthy children in Spain. Microb Drug Resist 8(4):321-327

25. Lidin-Janson G, Falsen E, Jodal U, Kaijser B, Lincoln K (1977) Characteristics of antibiotic-resistant Escherichia coli in the rectum of healthy school-children. J Med Microbiol 10(3):299-308 
26. Tramuta C, Robino P, Nucera D, Salvarani S, Banche G, Malabaila A, Nebbia P (2014) Molecular characterization and antimicrobial resistance of faecal and urinary Escherichia coli isolated from dogs and humans in Italy. Vet Ital 50(1):23-30

27. Skurnik D, Le Menac'h A, Zurakowski D, Mazel D, Courvalin P, Denamur E, Andremont A, Ruimy R (2005) Integron-associated antibiotic resistance and phylogenetic grouping of Escherichia coli isolates from healthy subjects free of recent antibiotic exposure. Antimicrob Agents Chemother 49(7):3062-3065

28. Tenaillon O, Skurnik D, Picard B, Denamur E (2010) The population genetics of commensal Escherichia coli. Nat Rev Microbiol 8(3):207-217

29. Johnson JR, Goullet P, Picard B, Moseley SL, Roberts PL, Stamm WE (1991) Association of carboxylesterase B electrophoretic pattern with presence and expression of urovirulence factor determinants and antimicrobial resistance among strains of Escherichia coli that cause urosepsis. Infect Immun 59(7):2311-2315

30. Le Gall T, Clermont O, Gouriou S, Picard B, Nassif X, Denamur E, Tenaillon O (2007) Extraintestinal virulence is a coincidental byproduct of commensalism in B2 phylogenetic group Escherichia coli strains. Mol Biol Evol 24(11):2373-2384

31. Peirano G, Pitout JD (2010) Molecular epidemiology of Escherichia coli producing CTX-M beta-lactamases: the worldwide emergence of clone ST131 O25:H4. Int J Antimicrob Agents 35(4):316-321

32. Novais A, Pires J, Ferreira H, Costa L, Montenegro C, Vuotto C, Donelli G, Coque TM, Peixe L (2012) Characterization of globally spread Escherichia coli ST131 isolates (1991 to 2010). Antimicrob Agents Chemother 56(7):3973-3976
33. Helldal L, Karami N, Florén K, Welinder-Olsson C, Moore ER, Åhrén C (2013) Shift of CTX-M genotypes has determined the increased prevalence of extended-spectrum beta-lactamaseproducing Escherichia coli in south-western Sweden. Clin Microbiol Infect 19(2):E87-E90

34. Cooke NM, Smith SG, Kelleher M, Rogers TR (2010) Major differences exist in frequencies of virulence factors and multidrug resistance between community and nosocomial Escherichia coli bloodstream isolates. J Clin Microbiol 48(4):1099-1104

35. Lavigne JP, Blanc-Potard AB, Bourg G, Moreau J, Chanal C, Bouziges N, O'Callaghan D, Sotto A (2006) Virulence genotype and nematode-killing properties of extra-intestinal Escherichia coli producing CTX-M beta-lactamases. Clin Microbiol Infect 12(12): 1199-1206

36. Lee S, Yu JK, Park K, Oh EJ, Kim SY, Park YJ (2010) Phylogenetic groups and virulence factors in pathogenic and commensal strains of Escherichia coli and their association with bla $a_{\mathrm{CTX}-\mathrm{M}}$. Ann Clin Lab Sci 40(4):361-367

37. Karami N, Hannoun C, Adlerberth I, Wold AE (2008) Colonization dynamics of ampicillin-resistant Escherichia coli in the infantile colonic microbiota. J Antimicrob Chemother 62(4):703-708

38. Karami N, Nowrouzian F, Adlerberth I, Wold AE (2006) Tetracycline resistance in Escherichia coli and persistence in the infantile colonic microbiota. Antimicrob Agents Chemother 50(1):156-161

39. Soto SM, Jimenez de Anta MT, Vila J (2006) Quinolones induce partial or total loss of pathogenicity islands in uropathogenic Escherichia coli by SOS-dependent or -independent pathways, respectively. Antimicrob Agents Chemother 50(2):649-653 